\title{
X-Ray Study of Variable Gamma-Ray Pulsar PSR J2021+4026
}

\author{
H. H. Wang ${ }^{1}$, J. Takata ${ }^{1}$ (1), C.-P. Hu ${ }^{2}$ (1), L. C. C. $\operatorname{Lin}^{3}$, and J. Zhao ${ }^{1}$ \\ ${ }^{1}$ School of Physics, Huazhong University of Science and Technology, Wuhan 430074, People's Republic of China; takata@ hust.edu.cn \\ ${ }^{2}$ Department of Physics, The University of Hong Kong, Pokfulam Road, Hong Kong \\ ${ }^{3}$ Department of Physics, UNIST, Ulsan 44919, Republic of Korea \\ Received 2017 October 11; revised 2018 January 11; accepted 2018 January 31; published 2018 March 28
}

\begin{abstract}
PSR J2021+4026 showed a sudden decrease in the gamma-ray emission at the glitch that occurred around 2011 October 16, and a relaxation of the flux to the pre-glitch state at around 2014 December. We report X-ray analysis results of the data observed by XMM-Newton on 2015 December 20 in the post-relaxation state. To examine any change in the X-ray emission, we compare the properties of the pulse profiles and spectra at the low gamma-ray flux state and at the post-relaxation state. The phase-averaged spectra for both states can be well described by a power-law component plus a blackbody component. The former is dominated by unpulsed emission and probably originated from the pulsar wind nebula as reported by Hui et al. The emission property of the blackbody component is consistent with the emission from the polar cap heated by the back-flow bombardment of the highenergy electrons or positrons that were accelerated in the magnetosphere. We found no significant change in the $\mathrm{X}$-ray emission properties between two states. We suggest that the change of the X-ray luminosity is at an order of $\sim 4 \%$, which is difficult to measure with the current observations. We model the observed X-ray light curve with the heated polar cap emission, and we speculate that the observed large pulsed fraction is owing to asymmetric magnetospheric structure.
\end{abstract}

Key words: methods: data analysis - pulsars: individual (J2021+4026) - stars: neutron - X-rays: stars

\section{Introduction}

A pulsar is a fast spinning and highly magnetized neutron star, which is a condensed star with an averaged mass density of $\sim 10^{14-15} \mathrm{~g} \mathrm{~cm}^{-3}$, and it is observable in radio to very highenergy $\mathrm{TeV}$ gamma-ray bands. Although the radio emission is the main window to investigate the timing properties of the pulsars, the all sky monitor of the Fermi Large Area Telescope (Fermi-LAT, Abdo et al. 2009a; Atwood et al. 2009; Ackermann et al. 2012), which is a space observatory launched in 2008, enables us to perform a long term survey of the pulsars in the gamma-ray bands. The Fermi-LAT has observed the gamma-ray emission from $>200$ pulsars $^{4,5}$ (Abdo et al. 2013). In particular, Fermi-LAT uncovered many new gamma-ray pulsars in the Cygnus region, and the most intriguing one among them is PSR J2021+4026. PSR J2021+4026 is an isolated pulsar that belongs to the Geminga-like pulsars (lacking radio-quiet emission with certain detections of pulsed detections in both the X-ray and gamma-ray bands; Lin 2016). It is also known as the first variable gamma-ray pulsar seen by the Fermi-LAT. It is associated with the supernova remnant G78.2+2.1 (Abdo et al. 2009b). This pulsar has a spin period of $P=265 \mathrm{~ms}$ and a spin-down rate of $\dot{P}=5.48 \times 10^{-14}$, corresponding to a characteristic age of $\tau_{c} \sim 77 \mathrm{kyr}$, a surface dipole field of $B_{d} \sim 4 \times 10^{12} \mathrm{G}$, and a spin-down power of $\dot{E} \sim 10^{35} \mathrm{erg} \mathrm{s}^{-1}$.

Allafort et al. (2013) reported results of a detailed analysis on the gamma-ray emission from PSR J2021+4026, and they found a glitch around MJD 55850 (2011 October 16) on a timescale shorter than one week. This glitch increased the spindown rate from $|\dot{f}|=(7.8 \pm 0.1) \times 10^{-13} \mathrm{~Hz} \mathrm{~s}^{-1}$ to $(8.1 \pm 0.1) \times 10^{-13} \mathrm{~Hz} \mathrm{~s}^{-1}$. Moreover, the glitch accompanied

\footnotetext{
4 https://confluence.slac.stanford.edu/display/GLAMCOG/Public +List + of +LAT-Detected +Gamma-Ray+Pulsars

5 https://fermi.gsfc.nasa.gov/ssc/data/access/lat/fl8y/
}

with (1) a decrease of flux $(>100 \mathrm{MeV})$ by $\sim 18 \%$, from $(8.33 \pm 0.08) \times 10^{-10} \mathrm{erg} \mathrm{cm}^{-2} \mathrm{~s}^{-1}$ to $(6.86 \pm 0.13) \times$ $10^{-10} \mathrm{erg} \mathrm{cm}^{-2} \mathrm{~s}^{-1},(2)$ a significant change in the pulse profile $(>5 \sigma)$, and (3) a marginal change in the gamma-ray spectrum $(<3 \sigma)$. Before the glitch, the pulse profile consisted of two strong peaks plus a small third peak in the bridge region. After the glitch, there was no evidence of the third peak. $\mathrm{Ng}$ et al. (2016) reanalyzed the Fermi-LAT data with a longer time span. They found that the flux drop caused by the glitch was a permanent-like effect, and the low gamma-ray flux state continued 3 years after the glitch. Zhao et al. (2017) reported the timing analysis of $\sim 8$ year Fermi-LAT data, and they found the relaxation at around 2014 December, where the spin-down rate and the gamma-ray emission returned to the state before the glitch. The pulse profile and spectrum after the relaxation are consistent with those before the glitch.

An unidentified X-ray source 2XMMJ202131.0+402645 had been investigated as a promising counterpart of PSR J2021 +4026 (Trepl et al. 2010; Weisskopf et al. 2011), and a deep observation was done by XMM-Newton (ESA: XMM-Newton $\mathrm{SOC}^{6}$ ) at MJD 56,028, when the pulsar stayed at the low gamma-ray flux state after the glitch. The detected spin frequency is consistent with the gamma-ray pulsation of PSR $\mathrm{J} 2021+4026$ at the same epoch, proving 2XMMJ202131.0 +402645 is indeed the counterpart of PSR J2021+4026 (Lin et al. 2013). The single broad pulse profile and the blackbody spectrum with an effective temperature of $k T_{B} \sim 0.25 \mathrm{keV}$ and radius $R_{\text {eff }} \sim 300 \mathrm{~m}$ imply that the X-ray emission came from the polar cap heated by the back-flow bombardment of the high-energy electrons or positrons that were accelerated in the magnetosphere.

Emission from the heated polar cap will be closely related to the gamma-ray emission process. In the outer gap model, for

\footnotetext{
6 "XMM-Newton Users Handbook," Issue 2.15, 2017.
} 


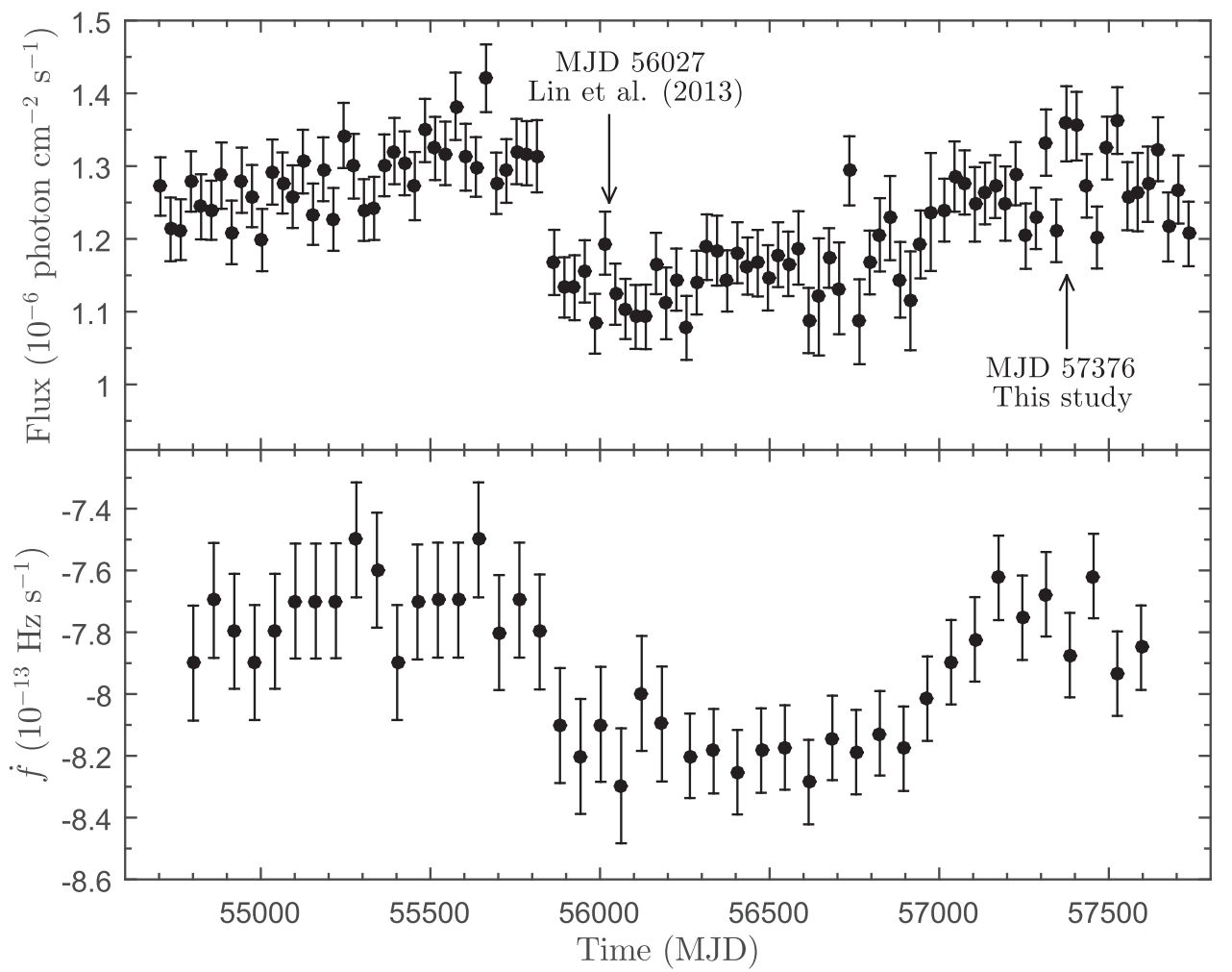

Figure 1. About an eight-year evolution of the gamma-ray flux (top) and the spin-down rate (bottom). The epochs of the two X-ray observations are indicted in the top panel. The figure is adopted from Zhao et al. (2017).

example, the pair-creation process inside the accelerator produces the electrons and positrons, and the electric field along the magnetic field separates the pairs, and results in the formation of the in-going and out-going particles (Zhang \& Cheng 1997). The created pairs inside the gap are accelerated to a Lorentz factor of $>10^{7}$ and emit the gamma-rays via the curvature radiation process. Half of the created pairs will return to and heat up the polar cap region. In this model, the flux of the observed X-ray emission will be proportional to the gamma-ray flux. Hence, it is expected that the state change in the spin-down rate/gamma-ray emission is also accompanied with the change in the observed X-ray emission. In this paper, we report the results of a new X-ray observation performed by $X M M$-Newton in the post-relaxation state, and compare the emission properties with the previous $\mathrm{X}$-ray properties observed in the low gamma-ray flux state (Section 2). In Section 3, we will discuss the X-ray light curve of the heated polar cap and the emission geometry.

\section{Data Analysis}

We analyze the archive data taken by XMM-Newton on 2015 December 20 (MJD 57,376, Obs. ID: 0763850101, PI: Razzano), which is about 3.7 years after the previous $X M M$ Newton observation performed in the low gamma-ray state (Lin et al. 2013), and about 1 year after the relaxation in 2014 December (Figure 1). This new observation was performed with a total exposure of $\sim 140 \mathrm{ks}$. The MOS1/2 CCDs were operated in the full-window mode (time resolution $2.6 \mathrm{~s}$ ), and PN CCD was operated in the small-window mode (time resolution $5.7 \mathrm{~ms}$ ). Only PN data enables the timing analysis of this pulsar. Event lists from the data are produced in the standard way using the most updated instrumental calibration and the emproc/epproc tasks of the XMM-Newton Science Analysis Software (XMMSAS, version 16.0.0). After filtering the events, which are potentially contaminated, the effective exposures are $134 \mathrm{ks}$ and $130 \mathrm{ks}$ for MOS1 and MOS2, $94 \mathrm{ks}$ for PN, respectively. A point source is significantly detected $(>10 \sigma)$ by XMMSAS task edetect_chain at the position of PSR J2021+4026. To perform the spectral and timing analyses, we extract EPIC data from circles with a radius of $20^{\prime \prime}$ centered at its nominal X-ray position (R.A., decl. $)=\left(20^{\mathrm{h}} 21^{\mathrm{m}} 30^{\mathrm{s}} .733\right.$, $\left.+40^{\circ} 26^{\prime} 46^{\prime \prime} 04\right)(\mathrm{J} 2000)$.

\subsection{Timing Analysis}

Our main purpose is to investigate the change in the pulsed $\mathrm{X}$-ray emission before and after the relaxation occurred at around 2014 December. Therefore, a timing analysis is crucial. Following Lin et al. (2013), we divide the PN data into three energy bands; $0.15-0.7 \mathrm{keV}, 0.7-2.0 \mathrm{keV}$, and $2.0-12 \mathrm{keV}$. For the timing analysis, the available photon number is 1399 counts for $0.15-0.7 \mathrm{keV}, 1170$ counts for $0.7-2.0 \mathrm{keV}$, and 724 counts for $2.0-12 \mathrm{keV}$. The arrival times of all the selected events are barycentric-corrected with the aforementioned X-ray position and the latest DE405 Earth ephemeris.

In the analysis, the pulsation is significantly detected only in $0.7-2.0 \mathrm{keV}$ energy bands, and no conclusive evidence of the pulsation is obtained in the energy bands of $0.15-0.7 \mathrm{keV}$ and 2.0-12 keV. Based on the Rayleigh test (Mardia 1972; Gibson et al. 1982) applied for $0.7-2.0 \mathrm{keV}$ observation, a significant peak is found at $f=3.7688991(2) \mathrm{Hz}$ with $Z_{1}^{2}=196$, where we assessed the uncertainty with Equation 6 (a) provided in Leahy (1987), using total duration of $\sim 100 \mathrm{ks}$. In $0.15-0.7 \mathrm{keV}$ and $2.0-12 \mathrm{keV}$ energy bands, the $\mathrm{X}$-ray pulsation is 


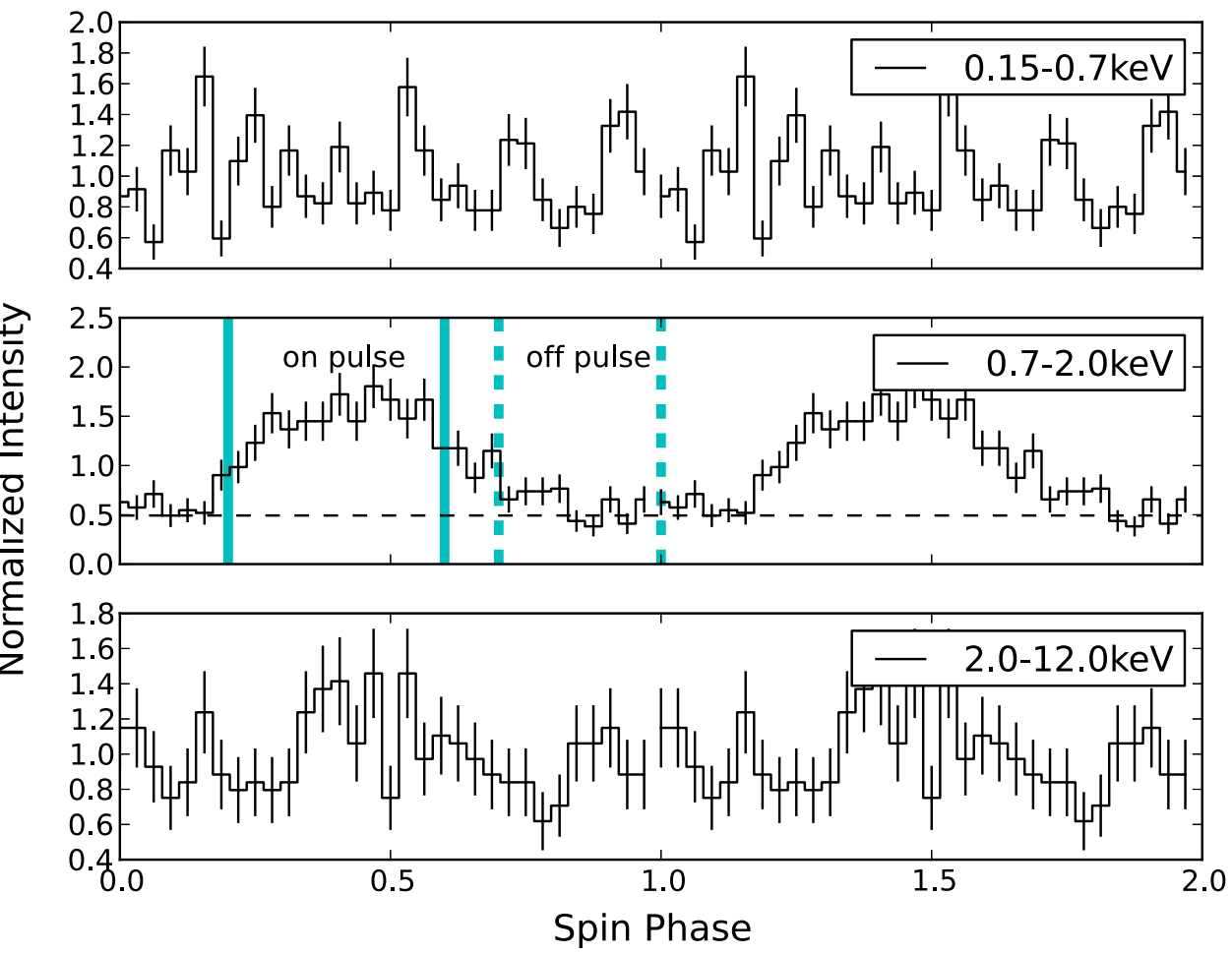

Figure 2. Energy dependent X-ray folded light curves of PSR J2021+4026 in the energy bands $0.15-0.7 \mathrm{keV}$ (upper), 0.7-2.0 keV (middle), and 2.0-12 keV (bottom). Two cycles are presented for clarity. In the middle panel, the vertical solid and dashed lines define the on-pulse and off-pulse phase, respectively, and the horizontal dashed line shows a background level determined by the nearby source-free region. We normalize the count so that the average intensity is unity, indicating the background emission occupies about $50 \%$ of the $0.7-2.0 \mathrm{keV}$ emission from the direction of the source.
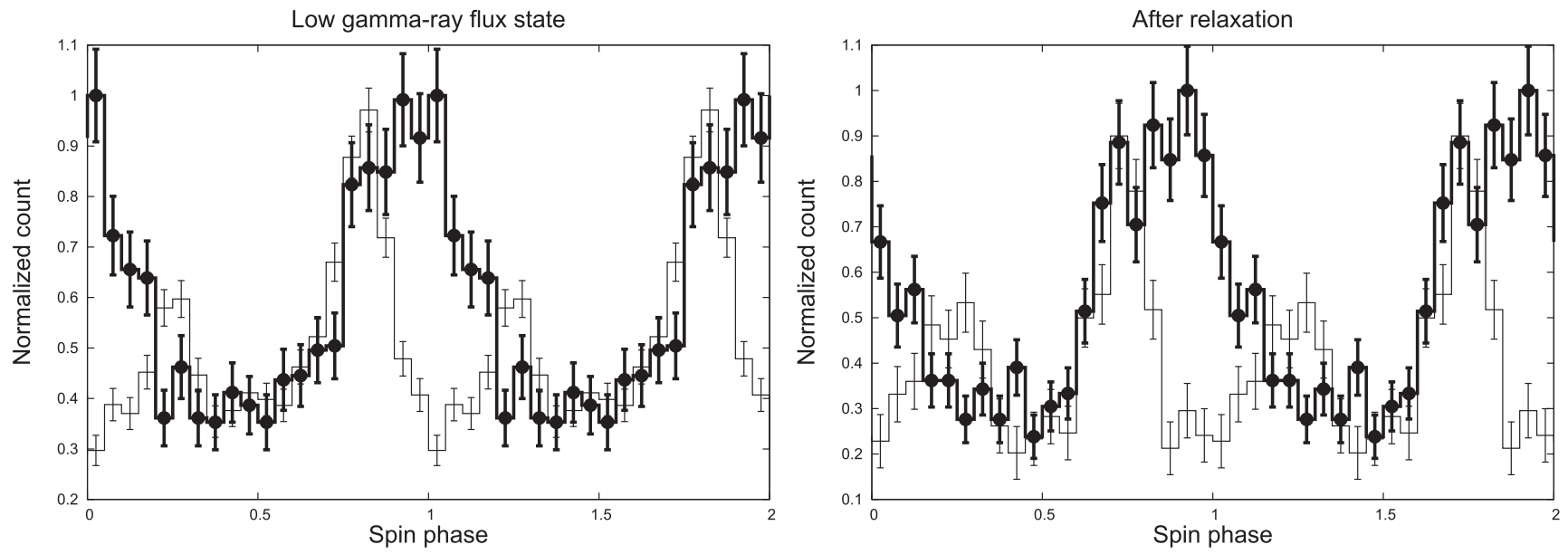

Figure 3. Folded light curves of PSR J2021 +4026 in $0.7-2.0 \mathrm{keV}$ (thick histograms) and $>0.1 \mathrm{GeV}$ (thin histograms) energy bands. Left panel: low gamma-ray flux state. Right panel: post-relaxation state.

insignificant with $Z_{1}^{2} \sim 5$. This spin frequency is consistent with that derived by the Fermi-LAT (Zhao et al. 2017).

Figure 2 shows the folded light curves in three energy bands; $0.15-0.7 \mathrm{keV}$ (top), $0.7-2.0 \mathrm{keV}$ (middle), and $2.0-12 \mathrm{keV}$ (bottom). In the figure, the folded light curve in $0.7-2.0 \mathrm{keV}$ bands shows a broad and single peak pulse profile. In Figure 3, we compare the light curves in $0.7-2.0 \mathrm{keV}$ energy bands in the low gamma-ray flux state (right panel) and in the post-relaxation state (left panel). Within the current quality of the data, no significant difference can be seen in the two pulse profiles.

In the X-ray light curve of the $0.7-2.0 \mathrm{keV}$ energy band (middle panel of Figure 2), we determine a background level (horizontal dashed line) with a nearby source-free region, and we can find that the background emission explains about 50\% of the emission from the direction of the source and it explains the emission at the offpulse phase. It is expected, therefore, that the pulse profile after subtracting the background has a large pulsed fraction, which is defined by $F=\left(f_{\max }-f_{\min }\right) /\left(f_{\max }+f_{\min }\right) \times 100 \%$ with $f_{\max }$ and $f_{\text {min }}$ being the maximum and minimum count rates, respectively. The large pulsed fraction $(F \sim 100 \%)$ can be used to constrain the emission geometry by modeling the light curve of the heated polar cap emission (Section 3).

Lin et al. (2013) compared the pulse phases in X-ray and gamma-ray bands in the low gamma-ray flux state, and found 
Table 1

Ephemeris of PSR J2021+4026

\begin{tabular}{lc}
\hline \hline Parameters & \\
\hline R.A. & $20^{\mathrm{h}} 21^{\mathrm{m}} 30^{\mathrm{s}} .733$ \\
Decl. & $+40^{\circ} 26^{\prime} 46^{\prime \prime} 04$ \\
Valid MJD range & $57,283 \sim 57,471$ \\
Pulse frequency, $f(\mathrm{~Hz})$ & $3.7688994400(7)$ \\
First derivative of pulse frequency, $\dot{f}\left(\mathrm{~s}^{-2}\right)$ & $-7.707(4) \times 10^{-13}$ \\
Second derivative of pulsar frequency, $\ddot{f}^{\left(\mathrm{s}^{-3}\right)}$ & $6.0(9) \times 10^{-22}$ \\
Third derivative of pulsar frequency, $\dddot{f}\left(\mathrm{~s}^{-4}\right)$ & $2(9) \times 10^{-29}$ \\
Epoch zero of the timing solution $(\mathrm{MJD})$ & 57,377 \\
rms timing residual $(\mu \mathrm{s})$ & 1677.862 \\
\hline
\end{tabular}

that the X-ray peak lags the stronger gamma-ray peak (the second peak) and proceeds after the smaller peak (the first peak). To investigate the phase relation between the X-ray and gamma-ray pulses in the post-relaxation state, we extract the Fermi-LAT data of about a half year (MJD 57,283-57,471) centered at the epoch of the X-ray observation, and create local ephemeris (Table 1). With the standard process for the data reduction using the Fermi-Scinece tools v10r0p5 package (Zhao et al. 2017 for more details of PSR J2021+4026), we assign the probability of each photon originating from the pulsar. After performing the gtbary task for barycentric time corrections to photon arrival times, we convert the arrival time to the pulsar spin phase using the local ephemeris created by Fermi-LAT data (i.e., Table 1).

Figure 3 summarizes the light curves of PSR J2021+4026 in the X-ray (thick histograms) and gamma-ray (thin histograms) using the same ephemeris reported in Lin et al. (2013) for the low gamma-ray flux state (left panel) and the ephemeris in Table 1 for the post-relaxation state (right panel). As we can see in the figure, the phase correlation between the X-ray peak and the gamma-ray peak in the post-relaxation state is very similar to that in the low gamma-ray flux state, that is, the $\mathrm{X}$-ray peak lags the stronger gamma-ray peak (the second peak) and proceeds after the smaller peak (the first peak). The crosscorrelation coefficient attains the maximum value at a phase lag of $\sim-0.14$ for both states. Hence, no significant change in the correlation of the X-ray and gamma-ray pulsations before and after the relaxation is found.

\subsection{Spectral Analysis}

\subsubsection{Phase-averaged Spectrum}

In order to further investigate the X-ray emission properties from this pulsar, we carry out the spectral analysis of all three EPIC cameras (MOS1/2 and PN), and we compare with the previous results given in the low gamma-ray flux state. We generate the spectra of MOS1/2 and $\mathrm{PN}$ from photons in the $0.15-12 \mathrm{keV}$ energy bands within a radius of $20^{\prime \prime}$ circle centered at the source. The background spectra are generated from a nearby region of the same size as the corresponding CCD. The response files are generated by the XMMSAS tasks rmfgen and arfgen. We group the channels so as to achieve the signal-to-noise ratio $\mathrm{S} / \mathrm{N}=3$ in each energy bin.

Figure 4 shows the phase-averaged spectrum in the postrelaxation state taken by all three cameras. To investigate any changes of the spectral properties in the low gamma-ray flux state and the post-relaxation state, we fit the data in the two states simultaneously using the XSPEC (version 12.9.1). Since the interstellar hydrogen is the main absorber of X-rays, we expect that the hydrogen column density $\left(N_{\mathrm{H}}\right)$ is not changed by the state change. In the analysis, therefore, we tie the column densities in the two data sets, and leave all other parameters free. First, we fit the data with the single-component model, that is, a single power law (PL) or a single blackbody (BB) radiation model. We found that the single-component model cannot provide appropriate fits; for the power-law model, the best-fit power-law index is very large $(\Gamma \sim 4)$ with $\chi^{2}=162$ for 121 degrees of freedom (dof), which is far steeper than that $(\Gamma \sim 1.5)$ predicted by the synchrotron radiation from the secondary electron/positron pairs (Cheng \& Zhang 1999). The blackbody model also provides an unacceptable fit with $\chi^{2}=205$ for 121 dof.

Since Lin et al. (2013) obtained a reasonable fit with the PL $+\mathrm{BB}$ model, we also apply the $\mathrm{PL}+\mathrm{BB}$ model for the simultaneous fitting. We found that the $\mathrm{PL}+\mathrm{BB}$ model can describe the data in the low gamma-ray flux state and the postrelaxation state simultaneously, and the best fitting parameters of the X-ray data in both states are consistent with each other within $1 \sigma$ error (Table 2); the observed spectra are well fitted with the photon index $\Gamma \sim 1-1.3$, the surface temperature of $k T_{B} \sim$ $0.25-0.3 \mathrm{keV}$ and the effective radius of $R_{\text {eff }} \sim 250-300 \mathrm{~m}$. The observed flux in the energy band of $0.2-12 \mathrm{keV}$ is dominated by the blackbody component. The result is consistent with that indicated in Lin et al. (2013), and the power-law component probably came from the pulsar wind nebula (Hui et al. 2015). Figure 5 shows the confidence contours on the plane determined by the photon index and the temperature. We can see in the figure that the distributions of the errors are very similar as well, suggesting no significant evolution of the spectral properties.

\subsection{Spectrum of the Pulsed Component}

We analyze the data in the low gamma-ray flux state and in the post-relaxation state, and we compare the spectra of the pulsed component for two states. According to Figure 2 of the post-relaxation state, we define the phase intervals of $0.2-0.6$ and $0.7-1.0$ as the "on-pulse" and "off-pulse" (i.e., DC level) components, respectively. We also use the same intervals in the light curves of the low gamma-ray flux state. The event files in each phase are extracted using xronwin and xselect, and the spectrum of the pulsed component is obtained by subtracting the spectrum of "off-pulse" phase from that of the "on-pulse" phase. Because of the limited photon counts, we grouped the channels so as to archive the signal-to-noise ratio $\mathrm{S} / \mathrm{N}=2$ in each energy bin. The generated spectra (Figure 6) are fitted by the single $\mathrm{BB}$ model, and its best-fit parameters (Table 3) are $k T_{B} \sim 0.26 \mathrm{keV}$ and $R_{\text {eff }} \sim 250 \mathrm{~m}$ at $d=1.5 \mathrm{kpc}$. The result in low gamma-ray flux state is fully consistent with the result in Hui et al. (2015). The single power-law model also provides an acceptable fit. However, the best-fit photon index is very large $(\Gamma \sim 3.8)$ with $\chi^{2}=8.41$ for 15 dof, again which is far steeper than the theoretical prediction $(\Gamma \sim 1.5)$. The two component $(\mathrm{BB}+\mathrm{PL})$ model fits the data with $k T_{B} \sim 0.19 \mathrm{keV}$, but the model cannot constrain the photon index. Based on our fitting presented in Table 3, no significant change is found in the pulsed spectra before and after relaxation of the state. 


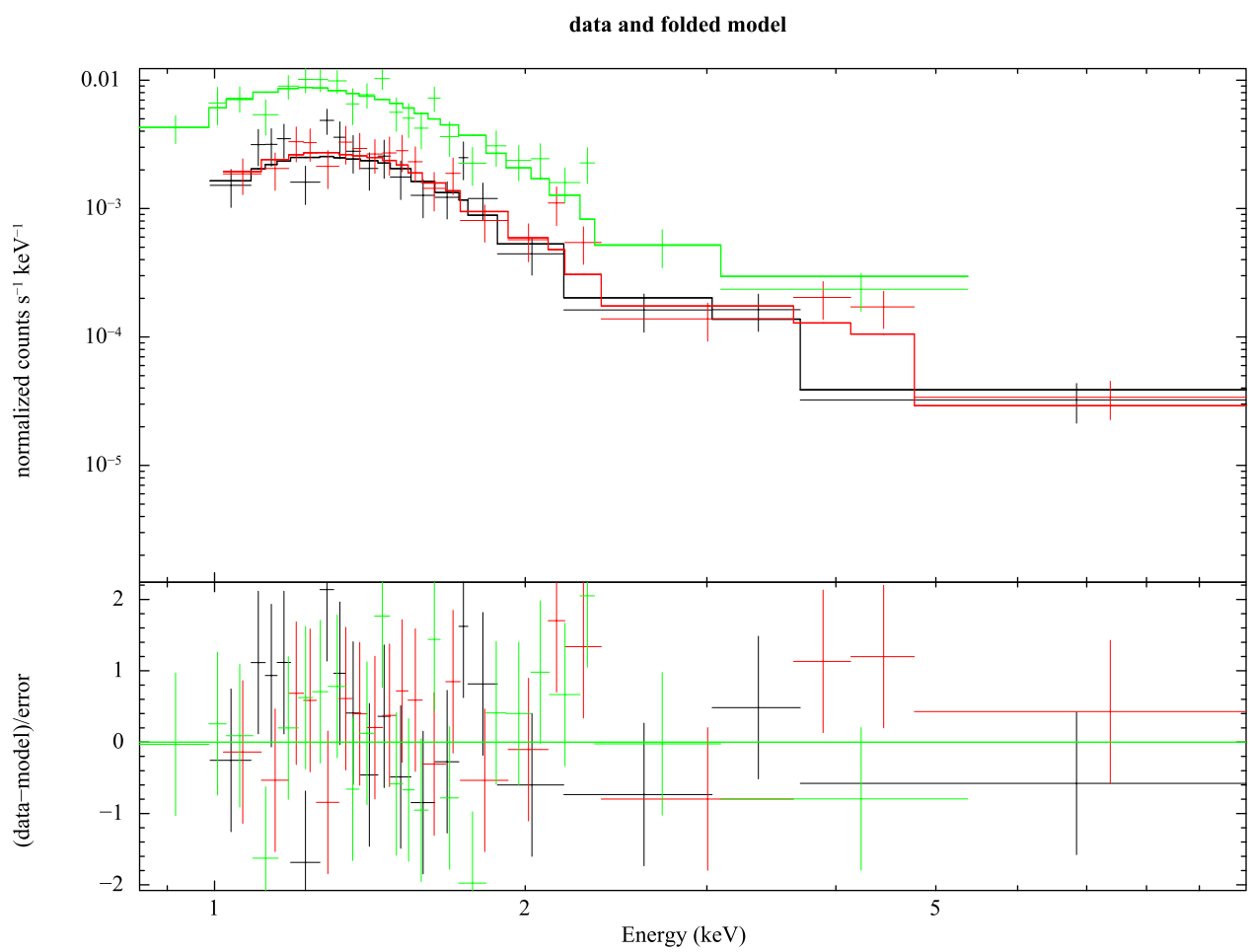

Figure 4. Phase-averaged spectrum of PSR J2021+4026 in 0.5-10 keV. The observed spectra with the PN (green spectrum) and MOS1/2 detectors (black and red spectra) are simultaneously fitted by an absorbed blackbody plus power-law model.

Table 2

Parameters of the Phase-averaged Spectra Determined in the Low Gamma-Ray Flux State (Middle Column) and in the Post-relaxation State (Third Column)

\begin{tabular}{lrr}
\hline \hline Parameters & MJD 56,028 & MJD 57,376 \\
\hline$N_{\mathrm{H}}\left(10^{22} \mathrm{~cm}^{-2}\right)$ & \multicolumn{1}{c}{$0.9_{-0.3}^{+0.4}$} & \\
Flux $_{b b}\left(10^{-13} \mathrm{erg} \mathrm{cm}^{-2} \mathrm{~s}^{-1}\right)^{\mathrm{a}}$ & $1.4_{-0.6}^{+1.4}$ & $1.6_{-0.7}^{+1.5}$ \\
Flux $_{p}\left(10^{-14} \mathrm{erg} \mathrm{cm}^{-2} \mathrm{~s}^{-1}\right)^{\mathrm{b}}$ & $3.5_{-0.7}^{+1.3}$ & $4.7_{-1.0}^{+1.4}$ \\
$k T_{B}(\mathrm{kev})$ & $0.21_{-0.03}^{+0.03}$ & $0.21_{-0.04}^{+0.04}$ \\
$R_{\text {eff }}(\mathrm{m})^{\mathrm{c}}$ & $272_{-251}^{+591}$ & $255_{-245}^{+622}$ \\
Photon index & $1.0_{-0.8}^{+0.7}$ & $1.3_{-0.9}^{+0.9}$ \\
$\chi^{2} /$ dof & \multicolumn{2}{c}{$105.89 / 114$} \\
\hline
\end{tabular}

Notes. The uncertainties of each of the spectral parameters are assessed in $1 \sigma$ for four parameters of interest for the multi-component model.

${ }^{a}$ Flux of the blackbody component in $0.2-12 \mathrm{keV}$.

${ }^{\mathrm{b}}$ Flux of the power-law component in $0.2-12 \mathrm{keV}$.

${ }^{\mathrm{c}}$ Effective radius at $d=1.5 \mathrm{kpc}$.

\section{Discussion and Summary}

\subsection{Emission from the Heated Polar Cap}

We have compared the properties of the X-ray emission observed at MJD 56,028 in the low gamma-ray flux state and at MJD 57,376 observed in the post-relaxation state. Although both spin-down rate and gamma-ray flux observed at the two epochs are different, our measurements find no significant change in any of the X-ray emission properties. The blackbody radiations in both states are measured with an effective radius $R_{\text {eff }} \sim 300 \mathrm{~m}$ at $d=1.5 \mathrm{kpc}$, which is comparable to the theoretical prediction of the polar cap size, $R_{\mathrm{pc}} \sim R_{\mathrm{NS}}\left(R_{\mathrm{NS}} / R_{\mathrm{lc}}\right)^{1 / 2} \sim 280 \mathrm{~m}$, where $R_{\mathrm{NS}}=$ $10^{6} \mathrm{~cm}$ is the neutron star radius, and $R_{\mathrm{lc}}=c P / 2 \pi$ is the light cylinder radius that can be derived from the spin period $(P)$ and the light speed $(c)$. As proposed in the previous studies (Lin et al.
2013; Hui et al. 2015), the observed X-ray emission likely originated from the polar cap heated by the bombardment of the high-energy electrons or positrons that were accelerated in an acceleration region.

Fermi-LAT found that the gamma-ray flux above the cutoff energy at around $\sim 3 \mathrm{GeV}$ decays slower than a pure exponential function (Abdo et al. 2013). This cutoff behavior favors the emissions from the outer magnetosphere (e.g., Arons 1983 for the slot gap model, Cheng et al. 1986a, 1986b for the outer gap model, and Spitkovsky 2006 for the current sheet), and it rules out the classical polar cap scenario (e.g., Daugherty \& Harding 1996), which predicted a super exponential cutoff feature in the $\mathrm{GeV}$ spectrum because of the magnetic pair-creation process. In the outer gap model (Cheng et al. 1986a, 1986b; Zhang \& Cheng 1997; Takata et al. 2010), for example, the luminosity of the gamma-ray from the accelerated electrons/positrons and of the heated polar cap are characterized by the so-called fractional gap width, $f_{\text {gap }}$, which is defined by the ratio of the angular size of the gap thickness measured on the stellar surface to the angular size of the polar cap (i.e., $\theta_{p}=\sqrt{R_{\mathrm{NS}} / R_{\mathrm{lc}}}$ ). The electrodynamics of the outer gap expects that the radiation power of the gamma-ray emission is of the order of

$$
L_{\gamma} \sim I_{\text {gap }} \times V_{\text {gap }} \sim f_{\text {gap }}^{3} L_{s d},
$$

where $I_{\text {gap }}$ and $V_{\text {gap }}$ are the electric current and potential drop along the magnetic field line, respectively. In the outer gap, they may be of the order of $I_{\text {gap }} \sim \pi f_{\text {gap }} B_{d} R_{\mathrm{NS}}^{3} /\left(P R_{\mathrm{lc}}\right)$ and $V_{\text {gap }}=$ $f_{\text {gap }}^{2} B_{d} R_{\mathrm{NS}}^{3} /\left(2 R_{\mathrm{lc}}^{2}\right)$, respectively, where $B_{d}$ denotes the surface magnetic dipole field. The gamma-ray emission from PSR J2021 $+4026\left(L_{s d} \sim 1.2 \times 10^{35} \mathrm{erg} \mathrm{s}^{-1}\right)$ is measured with a luminosity of $L_{\gamma} \sim 5 \times 10^{34}(d / 1.5 \mathrm{kpc})^{2}(\Delta \Omega / 3$ radian $) \mathrm{erg} \mathrm{s}^{-1}$, where $\Delta \Omega$ is the solid angle of the gamma-ray beam. The fraction gap 
Confidence contours: Chi-Squared

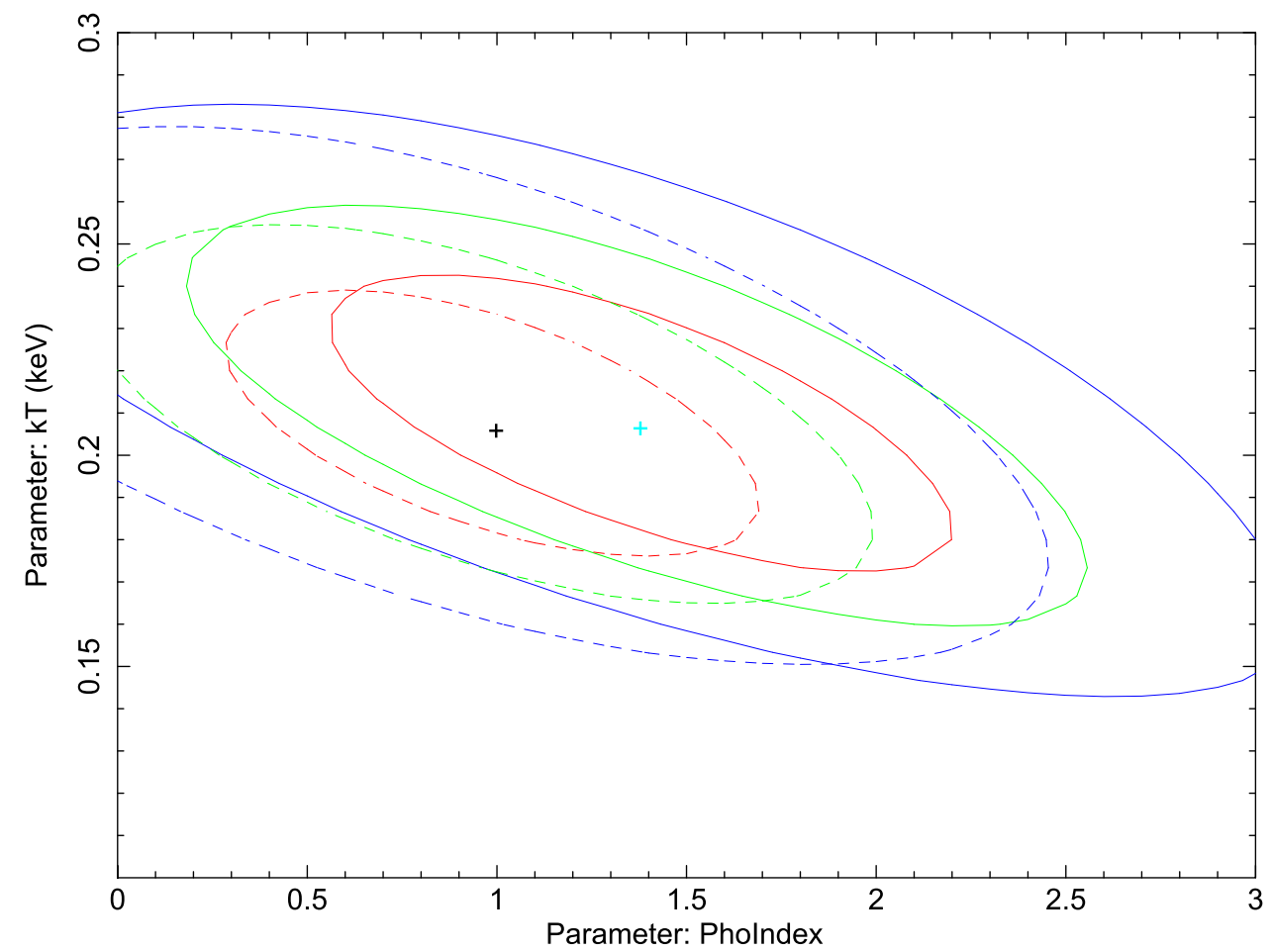

Figure 5. $1 \sigma, 2 \sigma$, and $3 \sigma$ error contours of the spectral parameters for the low gamma-ray flux state (dash line) and post-relaxation state (solid line). The black and blue cross symbols indicate the parameters providing the minimum $\chi^{2}$ for the low gamma-ray flux state and post-relaxation state, respectively.

thickness, therefore, is estimated as $f_{\text {gap }} \sim 0.75$, indicating that a large fraction of the spin-down power is converted into the highenergy radiation.

The electrons and positrons in the outer gap are separated by the electric field parallel to the magnetic field line, and half of the accelerated particles will be returned to the polar cap. The number of particles returned to the polar cap is of the order of

$$
\dot{N}_{e} \sim \frac{I_{\text {gap }}}{2 e} \sim 7 \times 10^{29} f_{\text {gap }} P^{-2} B_{12} \mathrm{~s}^{-1},
$$

where $B_{12}$ is the surface magnetic field in units of $10^{12} \mathrm{G}$. Because of the curvature radiation process during the travel from the inner boundary of the outer gap to the stellar surface, each return particle carries only $10.6 P^{1 / 3}$ ergs onto the stellar surface (Halpern \& Ruderman 1993; Zhang \& Cheng 1997). As a result, the luminosity of the X-rays from the heated polar cap region is estimated by

$$
L_{X} \sim 10.6 P^{1 / 3} \mathrm{erg} \cdot \dot{N}_{e} \sim 10^{31} f_{\text {gap }} B_{12} P^{-5 / 3} \mathrm{erg} \mathrm{s}^{-1},
$$

which gives the order of $L_{X} \sim 10^{32} \mathrm{erg} \mathrm{s}^{-1}$ for PSR J2021 +4026 . Since the X-ray emission from PSR J2021+4026 likely originated from the polar cap heated by the back-flow particles that were accelerated in the outer magnetosphere, it is expected that the state change in the spin-down rate/gamma-ray emission accompanies the change in the heated polar cap emission. In the low gamma-ray flux state, the spin-down rate was $\sim 4 \%$ higher than one in the pre-glitch and post-relaxation states. We speculate that the change in the magnitude of the global current caused the change in the spin-down rate at the glitch and at the relaxation, and that the current in the low gamma-ray flux state increased by $\delta I_{c} / I_{c} \sim 4 \%$. If we estimate the fractional gap thickness from $f_{\text {gap }}=\left(L_{\gamma} / L_{s d}\right)^{1 / 3}$, the observation implies that the fractional gap thickness decreased by $\delta f_{\text {gap }} / f_{\text {gap }} \sim-8 \%$. Since the $\mathrm{X}$-ray luminosity is proportional to $f_{\text {gap }} I_{c}$, the expected change in $L_{X}$ is found to be $\left|\delta L_{X} / L_{X}\right| \sim\left|\delta I_{c} / I_{c}+\delta f_{\text {gap }} / f_{\text {gap }}\right| \sim 4 \%$, which is smaller than the current uncertainty of our measurement $(>10 \%)$. Hence it is difficult to measure the change of the X-ray emission before and after the relaxation with the current observations.

\subsection{X-Ray/Gamma-Ray Light Curve Model}

As we have discussed above, the X-ray emission likely originated from the heated polar cap region, and the $\mathrm{GeV}$ gamma-ray may be produced in the outer magnetosphere. The $\mathrm{X}$-ray peak leading the first peak in the gamma-ray pulse profile will also be consistent with the GeV gamma-ray emission from the outer magnetosphere (e.g., Romani \& Yadigaroglu 1995). The gamma-ray emission from PSR J2021+4026 is observed with a double peak in the light curve separated by about a halfspin phase. Trepl et al. (2010) interpreted the phase separation with the outer gap accelerator model and expected the viewing angle of $\sim 90^{\circ}$ measured from the rotation axis. Hui et al. (2015) assumed an orthogonal rotator with a single pole contribution to fit the X-ray light curve. Since the previous studies did not discuss in detail the phase relation between the gamma-ray pulse and X-ray pulse for PSR J2021+4026, we compare the observed $\mathrm{X}$-ray/GeV light curves with the theoretical model.

Since the radius of the polar cap region of PSR J2021+4026 is of the order of 200-300 m, which is much smaller than the neutron star size, we can safely ignore the size of the polar cap region, and assume the point-like hot spots at the magnetic poles. 


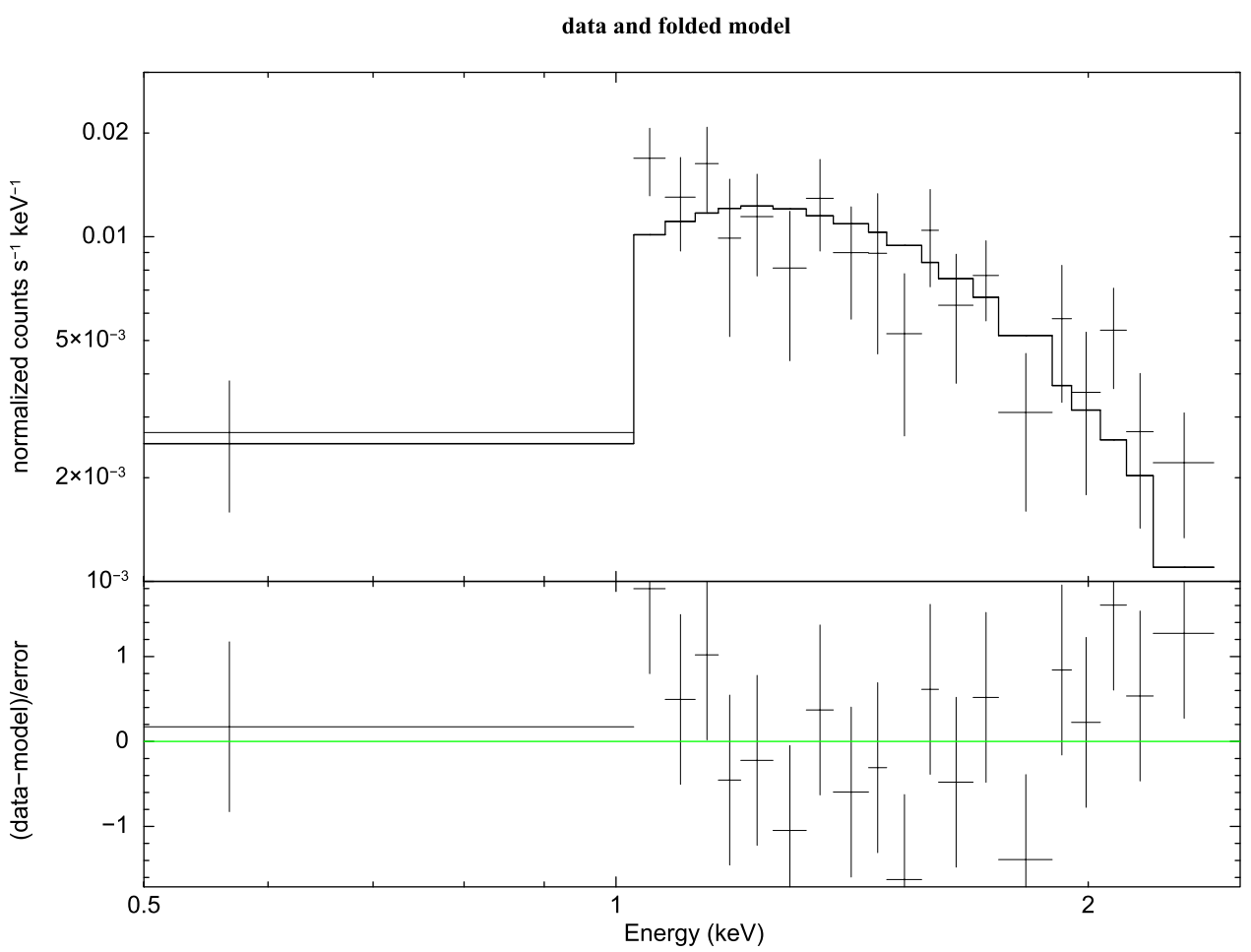

Figure 6. Pulsed spectrum of PSR J2021+4026 in the post-relaxation state. This spectrum is obtained by subtracting the data of the off-pulse phase from the on-pulse phase and provides the best fit for a single blackbody model in the energy band of $0.2-7 \mathrm{keV}$. The lower panel demonstrates the $\chi^{2}$ fit statistic.

Table 3

Parameters of the Pulsed Spectra in the Low Gamma-Ray Flux State (Middle Column) and in the Post-relaxation State (Third Column)

\begin{tabular}{|c|c|c|}
\hline Parameter & MJD 56,028 & MJD 57,376 \\
\hline Flux $\left(10^{13} \mathrm{erg} \mathrm{cm}^{-2} \mathrm{~s}^{-1}\right)^{\mathrm{a}}$ & $1.3_{-0.3}^{0.3}$ & $1.0_{-0.3}^{+0.4}$ \\
\hline$k T_{B}(\mathrm{keV})$ & $0.27_{-0.03}^{+0.04}$ & $0.27_{-0.04}^{+0.05}$ \\
\hline$R_{\text {eff }}(\mathrm{m})^{\mathrm{b}}$ & $234_{-168}^{+240}$ & $214_{-163}^{+258}$ \\
\hline$\chi^{2} /$ dof & \multicolumn{2}{|c|}{$17.13 / 17$} \\
\hline
\end{tabular}

Notes. The hydrogen column density is fixed at $N_{\mathrm{H}}=7 \times 10^{21} \mathrm{~cm}^{-2}$.

${ }^{\mathrm{a}}$ Flux in $0.2-12 \mathrm{keV}$.

${ }^{\mathrm{b}}$ Effective radius at $d=1.5 \mathrm{kpc}$.

The theory including gravitational light bending for the hotspot emission on the neutron star surface has been developed in the previous studies (Pechenick et al. 1983; Beloborodov 2002; Bogdanov et al. 2007; Bogdanov 2016). In this paper, we apply an approximation relation (Beloborodov 2002; Bogdanov 2016),

$$
\cos \psi \sim \frac{\cos \theta-R_{s} / R_{\mathrm{NS}}}{1-R_{S} / R_{\mathrm{NS}}}
$$

where $\psi$ and $\theta$ are the escape direction and the local angle of the emission direction, respectively. In addition $R_{s}=2 G M_{\mathrm{NS}} / c^{2}$, and $M_{\mathrm{NS}}$ is the mass of the neutron star. Such a formula can be used for $R_{\mathrm{NS}} / R_{S}>2$. In this paper, we present a result with $R_{\mathrm{NS}} / R_{s}$ that is $\sim 2.35$ for typical $M_{\mathrm{NS}}=1.4 M_{\odot}$ and $R_{\mathrm{NS}}=$ $10 \mathrm{~km}$. The escape angle $\psi$ is related to the spin phase as

$$
\cos \psi(t)=\sin \alpha \sin \zeta \cos \left(2 \pi \frac{t}{P}\right)+\cos \alpha \cos \zeta
$$

where $\alpha$ and $\zeta$ are the angles of the magnetic axis and the line of sight measured from the spin axis.

We assume that the observed count at each spin phase in the light curve is proportional to $I_{0}(\theta) \cos \theta$, where $I_{0}(\theta)$ is the local intensity. For the isotropic emission, $I_{0}(\theta)=$ constant, the previous studies (e.g., Beloborodov 2002; Bogdanov 2016) demonstrated that the emissions from the two PCs, which are equally heated up, make the pulse profile with a small pulsed fraction $F \sim 10 \%$ (see also Figure 7). This is because the light bending effect allows us to measure the emission from the backside of a neutron star and because the observer can almost always see the emission from two poles. A neutron star atmosphere model with a strong magnetic field has been developed by the previous studies (e.g., Pavlov et al. 1994; Zavlin et al. 1995; Ho \& Lai 2001, 2003; Zane \& Turolla 2006). It is discussed that the neutron star atmosphere with a strong magnetic field makes the emission highly anisotropic, and the higher intensity emerges along the magnetic field. Zavlin et al. (1995) demonstrate that the atmosphere with a strong magnetic field can generate the light curve with a larger pulsed fraction and a shaper pulse width.

To compare the atmosphere-model light curve with the observation of PSR J2021+4026, we refer to the results of Zavlin et al. (1995), who calculate the angular distribution, $I(\theta)$, with the pulsar parameters of $k_{B} T_{\text {eff }} \sim 0.25 \mathrm{keV}$ and $B_{d} \sim$ $1.2 \times 10^{12} \mathrm{G}$. Since their temperature of the heated polar cap is similar to that of PSR J2021+4026 ( $\left.k T_{\text {eff }} \sim 0.21 \mathrm{keV}\right)$, we may expect that their results can represent the case of PSR J2021 +4026. Pavlov et al. (1994) compare the calculated angular distributions of the intensity for the nonmagnetic case $B_{d}=0$ and the magnetic case $B_{d}=1.18 \times 10^{12} \mathrm{G}$. From their results, we may expect that a slight difference in the dipole magnetic field $\left(B_{d} \sim 4 \times 10^{12} \mathrm{G}\right.$ for PSR J2021+4026 comparing with 

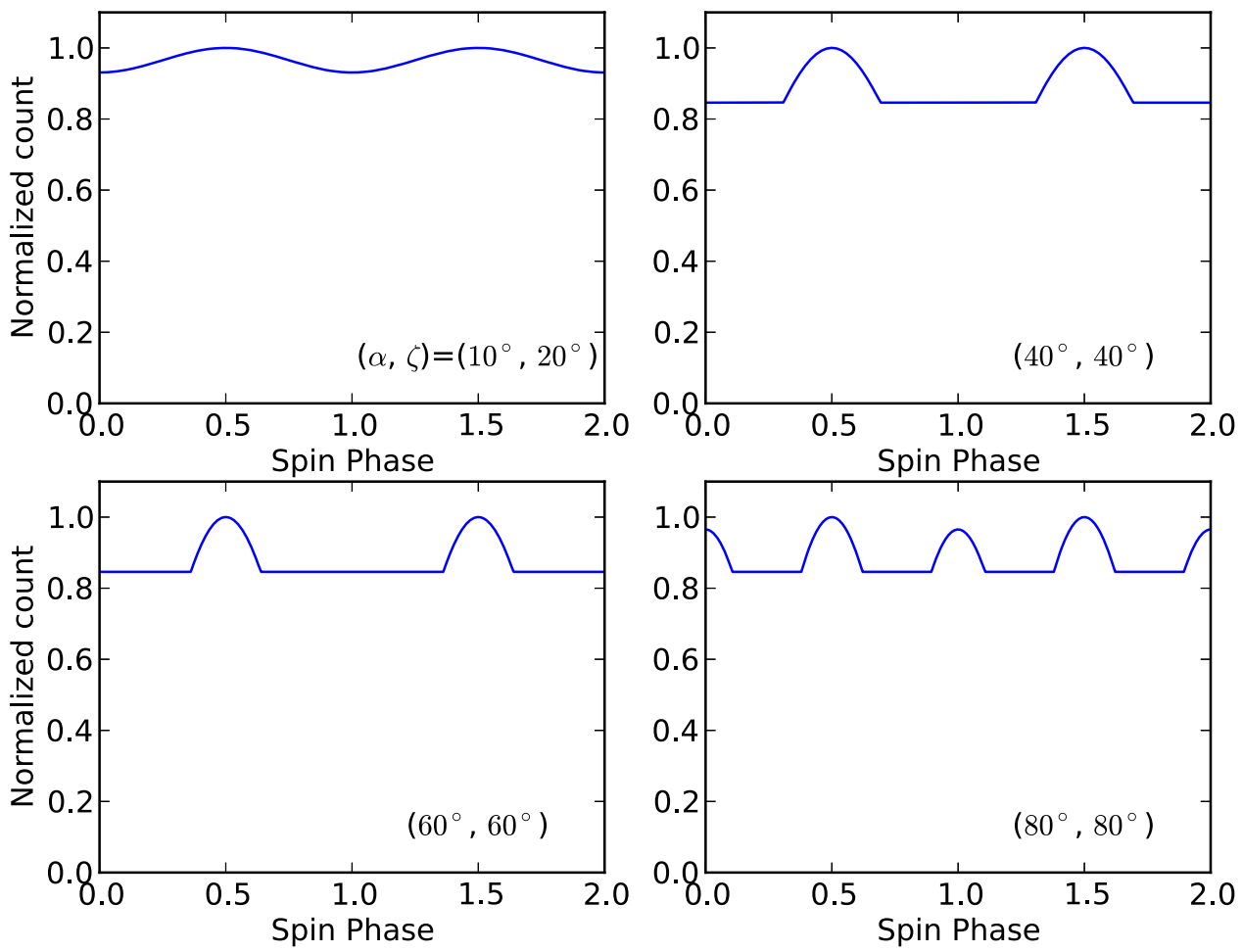

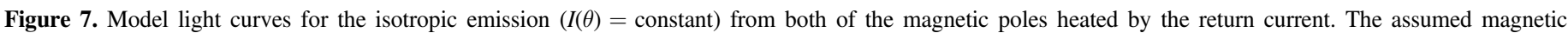

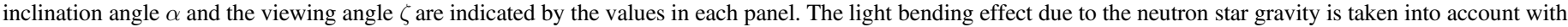
$R_{\mathrm{NS}} / R_{s}=2.35$ (Beloborodov 2002; Bogdanov 2016).
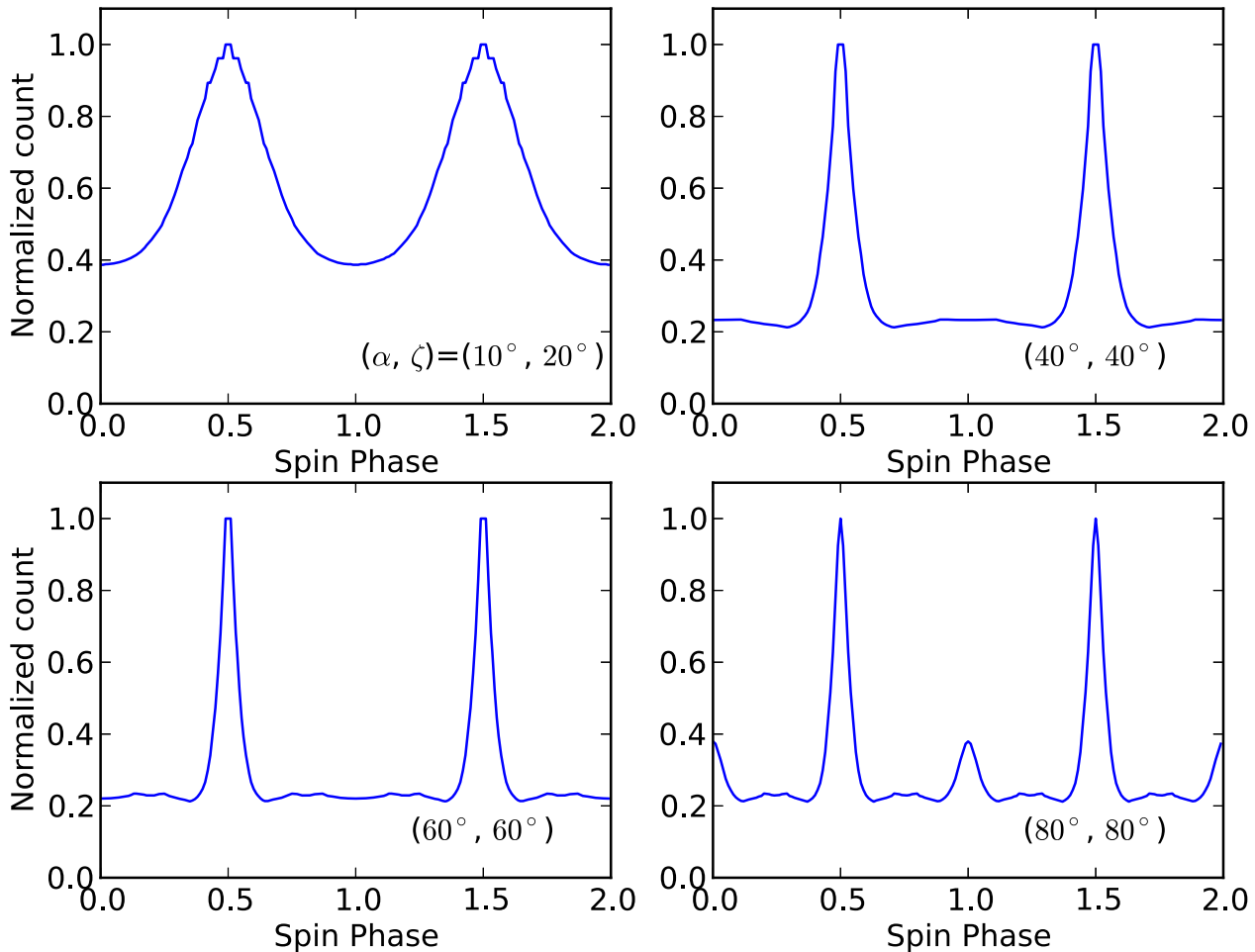

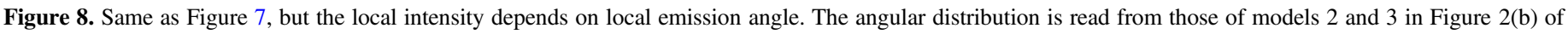
Zavlin et al. (1995)

$B_{s} \sim 1.2 \times 10^{12} \mathrm{G}$ of Zavlin et al. 1995) does not significantly change the angular distribution. Zavlin et al. (1995) present the angular distributions for the photon energy $E_{0}=0.18,1.12$, and $2.47 \mathrm{keV}$ (Figure 2 in Zavlin et al. 1995). Since the significant pulsation of PSR J2021+4026 is detected in
0.7-2 keV energy bands, we read the angular distribution of $E=1.12 \mathrm{keV}$ from Figure 2(b) in Zavlin et al. (1995). Finally, Zavlin et al. (1995) also show that the angular distribution is less dependent on the neutron star model, that is, $R_{\mathrm{NS}} / R_{s}$. In this paper, therefore, we refer their result of $R_{\mathrm{NS}} / R_{s}=2.418$ 


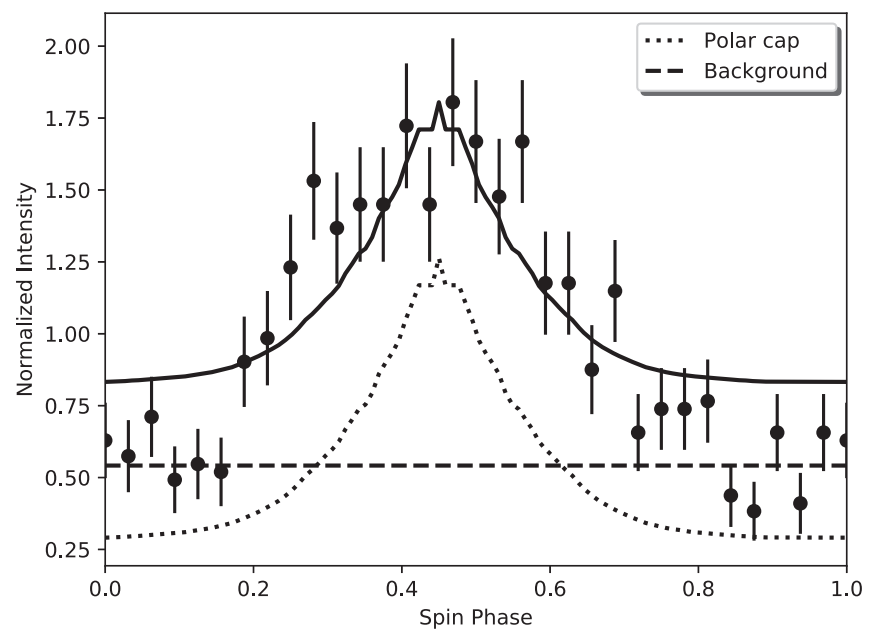

Figure 9. Comparing between the model and observed (0.7-2 keV) light curves. For the heated polar cap emission (dotted line), the angular distribution of the intensity is approximated by Figure 2(b) of Zavlin et al. (1995). The background level (dashed line) is determined by the nearby source-free region. The model result is for $\alpha \sim 16^{\circ}$ and $\zeta \sim 20^{\circ}$, which is determined by the minimum chi-square.

(model 2 in Zavlin et al. 1995). Figure 8 summarizes the model light curves for the different set of the inclination angle and viewing angle. By comparing Figures 7 with 8 , we can see that the atmosphere with the magnetic field makes the light curve with a larger pulsed fraction $(F \sim 65 \%)$ and a shaper pulse width, as demonstrated in the previous studies (Pavlov et al. 1994; Zavlin et al. 1995).

Figure 9 compares the model light curve and the observed $0.7-1.2 \mathrm{keV}$ light curve; in the model, we assume that $50 \%$ of the total emission (solid line) is contributed by the background (dashed line), as indicated by the observed $0.7-2.0 \mathrm{keV}$ emission (Figure 2). We determine the inclination angle $\alpha \sim 16^{\circ}$ and the viewing angle $\zeta \sim 20^{\circ}$ to minimize the chisquare with the central points of the data. Within the current simple treatment of the angular distribution, we find that although the size of the observational error is relatively large, the pulsed fraction predicted in the model light curve is smaller than that of the observation, as Figure 9 indicates. The pulsed fraction indeed depends on many unknown parameters (e.g., hotspot size, viewing geometry, beaming effect, and $R_{\mathrm{NS}} / R_{s}$ ). For example, we can expect that the pulsed fraction decreases with the increase of the hotspot size and with the decrease of the ratio $R_{\mathrm{NS}} / R_{s}$. The pulsed fraction tends to increase with the increase of the ratio $R_{\mathrm{NS}} / R_{s}$. The observed pulsed fraction $(F \sim 100 \%)$, however, can be explained by an unrealistically large ratio $R_{\mathrm{NS}} / R_{s}>5$ with the current beaming effect. With a reasonable ratio $R_{\mathrm{NS}} / R_{s}$, a larger beaming effect will be required to give rise to the observed pulsed fraction. A fine tuning of the parameters is required to explain the observed $\mathrm{X}$-ray light curve if two poles are equally heated up.

To obtain a more robust conclusion, it will be required to calculate the light curve integrated by the photon energy and with the magnetic field strength of PSR J2021+4026, which will be done in subsequent studies. We may argue, on the other hand, that if both poles are equally heated up by the return currents, the resultant pulsed fraction would be smaller than that observed for PSR J2021+4026, as Figure 9 indicates. Since it would not be necessary that the magnetosphere is symmetric, the north and south poles could have different temperatures, and the resultant light curve has a large pulsed fraction. We speculate that the asymmetry could be introduced by asymmetry of the magnetic field structure around the polar caps. It is argued that near the stellar surface, the magnetic field configuration is not dominated by a dipole field (Ruderman \& Cheng 1988; Ruderman 1991). Higher order multipole field configuration is likely, and the strength of the multipole field can be $1 \sim 3$ orders of magnitude larger than the global dipole field. This could also affect the structure and magnetic paircreation process of the polar cap accelerator. For example, Timokhin \& Harding (2015) point out that the multiplicity of the pair-creation cascade at the polar cap accelerator is sensitive to the curvature and strength of the magnetic field. Moreover, Harding \& Muslimov (2011a, 2011b) argue that even a small distortion of the dipole field and/or the offset of the polar cap from the dipole axis can greatly enhance the accelerating electric field and the resultant multiplicity of the pairs. It is expected therefore that an asymmetry of the magnetic field of the two polar caps causes the asymmetry of the polar cap accelerators. If the polar cap accelerator supplies the return current to heat up the polar cap, the temperatures of the two polar caps could be different.

The return particles can also be supplied by the accelerator around the light cylinder, which is probably the emission site of the observed GeV gamma-rays. For example, the outer gap accelerator is a possible region to supply the returning particles. It has been argued that the outer gap structure is sensitively controlled by the electrons or positrons that enter into the gap from the outside along the magnetic field lines (Hirotani \& Shibata 2001; Takata et al. 2016). Moreover, the outer gap will be quenched (or less luminous), if the particles with a super Goldreich-Julian rate are supplied from the polar cap region to the outer gap accelerator. If the multipole fields in the south and north poles could be an asymmetric configuration, the asymmetric pair-creation process at the two polar caps could make one outer gap, connecting to one pole, less active than that connecting to the other pole.

The high-energy emission in the outer magnetosphere has been discussed with the slot gap model (e.g., Arons 1983; Harding et al. 2008; Harding \& Kalapotharakos 2015), the outer gap model (e.g., Cheng et al. 1986a, 1986b; Takata et al. 2011), and the current sheet of the force-free or dissipative pulsar magnetosphere model (e.g., Spitkovsky 2006; Kalapotharakos et al. 2014, 2017; Cerutti \& Beloborodov 2017). These models predict that a larger viewing angle of $\zeta \sim 90^{\circ}$ is preferred to explain the double peak structure of the $\mathrm{GeV}$ emission with the peak separation of $\sim 0.5$ phase for PSR J2021+4026 (Takata et al. 2011; Kalapotharakos et al. 2014). With a larger viewing angle, on the other hand, the emission from two polar caps that are equally heated up will make a double peak structure in the X-ray light curve, as demonstrated in Figures 7 and 8, which is inconsistent with the observation. This also motivates us to speculate that one polar cap is less active.

Figure 11 shows the model light curves of the X-ray from the heated polar cap and of the gamma-ray from the outer gap accelerator by assuming that only one magnetic hemisphere is active, as illustrated by Figure 10; we assume $\alpha=60^{\circ}$ and $\zeta=85^{\circ}$, and the outer gap in the north hemisphere (left panel) or the south hemisphere (right panel) is active. As in Figure 8, we refer to the angular distribution of the local emission of the polar cap from Zavlin et al. (1995). We find in the figure that the single pole model can produce the single peak with a large pulsed fraction, and it is consistent with the observation. To 

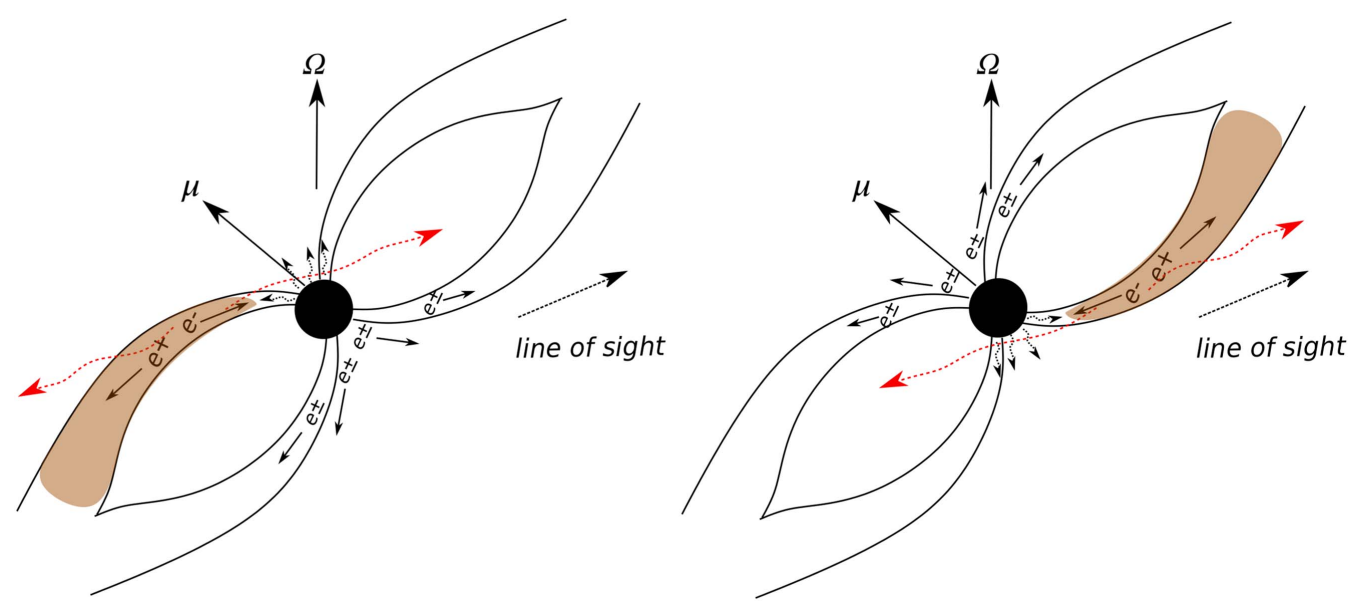

Figure 10. Possible geometry for PSR J2021+4026. The charge separated pairs in the outer gap produce inwardly and outwardly propagating gamma-rays (red arrows), and the return particles heat up the polar cap region. The X-ray pulse profile indicates that only the single pole is heated up by the return current from the outer gap, and the outer gap in other hemisphere is quenched by the copious pairs from the polar cap region. The figure assumes $\zeta<90^{\circ}$ for the observer viewing angle, and only the outer gap connecting to the north pole (left panel) or south pole (right panel) is active. The double peak structure of the GeV light curve indicates that the line of sight is nearly $90^{\circ}$.
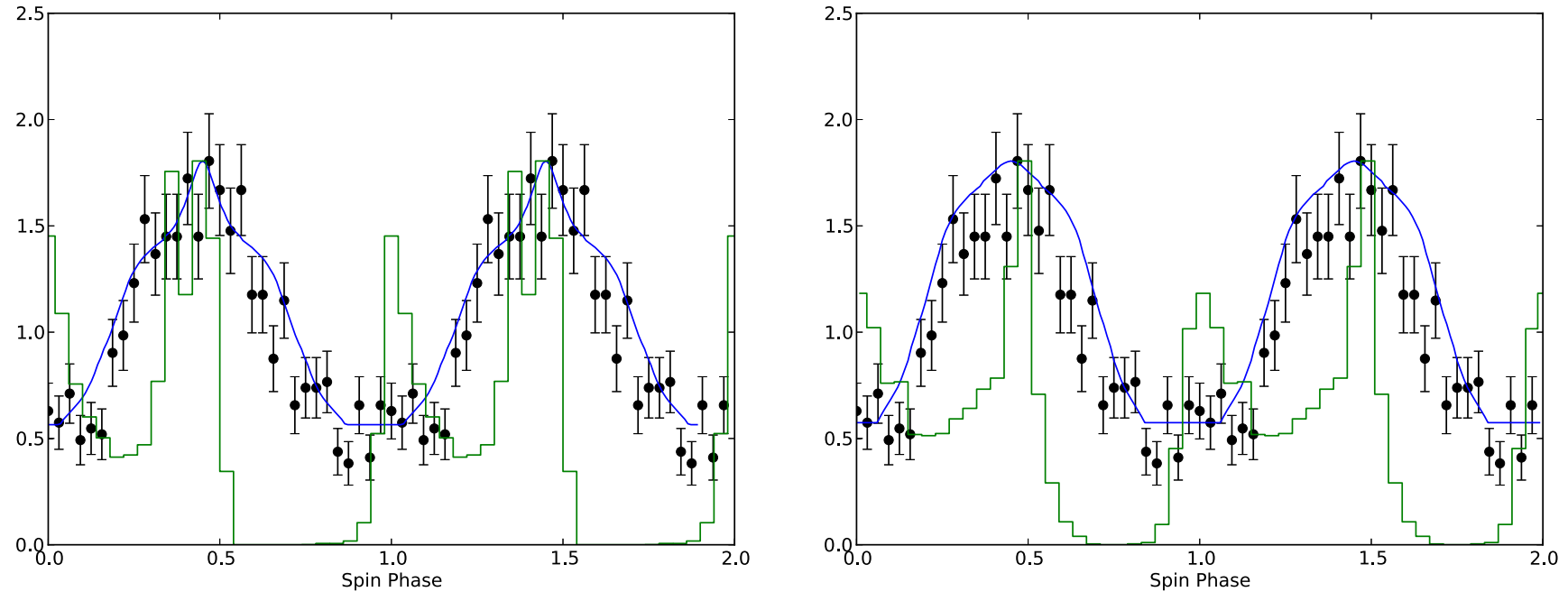

Figure 11. Model X-ray (blue curve) and GeV (green histogram) light curves of PSR J2021+4026. The emission geometry is the same as in Figure 10, and the left (or right) panel assumes that only the outer gap connecting to north pole (or south pole) is active. The results are for the inclination angle $\alpha=60^{\circ}$ and the viewing angle $\zeta=85^{\circ}$, which are chosen to explain both observed phase-separation of the gamma-ray peaks and the pulsed fraction of the X-ray light curve.

investigate the phase relation between the gamma-ray pulse and the X-ray pulse, we apply a simple outer gap model based on Wang et al. (2010), and consider both outwardly and inwardly propagating gamma-rays from the outer gap. In Figure 11, we can see that the second peak of the gamma-ray light curve (green lines) is almost aligned with the X-ray peak, and the first peak is located at the minimum of the X-ray light curve. These features are roughly consistent with the observed correlation in Figure 3. The current observation indicates that the X-ray peak shifts from the second peak of the gamma-ray light curve by the $\sim 0.14$ phase (Section 2.1). This may indicate that realistic magnetic field geometry at the polar cap or at the outer gap may be different from the dipole field.

In summary, we have examined the properties of the X-ray emission from the variable gamma-ray pulsar, PSR J2021 +4026 , in the low gamma-ray flux state and the post-relaxation state. The X-ray emission from both states are well described by a power law plus the blackbody radiation. The former is probably unpulsed and originated from the pulsar wind nebula (Hui et al. 2015). The emission property of the blackbody component is consistent with the emission from the polar cap heated by the back-flow bombardment of the high-energy electrons or positrons that were accelerated in the magnetosphere. We found no significant change in the X-ray emission properties at both states. The X-ray pulse profile could be fitted better by the emission from one pole rather than two poles, which suggests an asymmetric magnetosphere.

We express our appreciation to an anonymous referee for useful comments and suggestions. We thank K.S. Cheng, C.-Y. $\mathrm{Ng}$, C.Y. Hui, A.K. Kong, and P.H. Tam for useful discussions. W.H.H. and J.T. are supported by NSFC grants of the Chinese Government under 11573010, U1631103, and 11661161010. L.L.C.C. is supported by the National Research Foundation of Korea through grant 2016R1A5A1013277.

\section{ORCID iDs}

J. Takata (iD https://orcid.org/0000-0002-8731-0129 C.-P. Hu (i) https://orcid.org/0000-0001-8551-2002 


\section{References}

Abdo, A. A., Ackermann, M., Ajello, M., et al. 2009a, APh, 32, 193 Abdo, A. A., Ackermann, M., Ajello, M., et al. 2009b, ApJS, 183, 46 Abdo, A. A., Ajello, M., Allafort, A., et al. 2013, ApJS, 208, 17 Ackermann, M., Ajello, M., Albert, A., et al. 2012, ApJS, 203, 4 Allafort, A., Baldini, L., Ballet, J., et al. 2013, ApJL, 777, L2 Arons, J. 1983, ApJ, 266, 215

Atwood, W. B., Abdo, A. A., Ackermann, M., et al. 2009, ApJ, 697, 1071 Beloborodov, A. M. 2002, ApJL, 566, 85

Bogdanov, S. 2016, EPJA, 52, 37

Bogdanov, S., Rybicki, G. B., \& Grindlay, J. E. 2007, ApJ, 670, 668

Cerutti, B., \& Beloborodov, A. M. 2017, SSRv, 207, 111

Cheng, K. S., Ho, C., \& Ruderman, M. 1986a, ApJ, 300, 500

Cheng, K. S., Ho, C., \& Ruderman, M. 1986b, ApJ, 300, 522

Cheng, K. S., \& Zhang, L. 1999, ApJ, 515, 337

Daugherty, J. K., \& Harding, A. K. 1996, ApJ, 458, 278

Gibson, A. I., Harrison, A. B., Kirkman, I. W., et al. 1982, Natur, 296, 833

Halpern, J. P., \& Ruderman, M. 1993, ApJ, 415, 286

Harding, A. K., \& Kalapotharakos, C. 2015, ApJ, 811, 63

Harding, A. K., \& Muslimov, A. G. 2011a, ApJL, 726, 10

Harding, A. K., \& Muslimov, A. G. 2011b, ApJ, 743, 181

Harding, A. K., Stern, J. V., Dyks, J., \& Frackowiak, M. 2008, ApJ, 680, 1378

Hirotani, K., \& Shibata, S. 2001, ApJ, 558, 216

Ho, W. C. G., \& Lai, D. 2001, MNRAS, 327, 1081

Ho, W. C. G., \& Lai, D. 2003, MNRAS, 338, 233
Hui, C. Y., Seo, K. A., Lin, L. C. C., et al. 2015, ApJ, 799, 76

Kalapotharakos, C., Harding, A. K., \& Kazanas, D. 2014, ApJ, 793, 97

Kalapotharakos, C., Harding, A. K., Kazanas, D., \& Brambilla, G. 2017, ApJ, 842,80

Leahy, D. A. 1987, A\&A, 180, 275

Lin, L. C. C. 2016, JASS, 33, 147

Lin, L. C. C., Hu, C. Y., Hu, C. P., et al. 2013, ApJL, 770, L9

Mardia, K. V. 1972, Statistics of Direction Data (New York: Academic)

Ng, C. W., Takata, J., Cheng, K. S., et al. 2016, ApJ, 825, 18

Pavlov, G. G., Shibanov, Y. A., Ventura, J., \& Zavlin, V. E. 1994, A\&A, 289, 837

Pechenick, K. R., Ftaclas, C., \& Cohen, J. M. 1983, ApJ, 274, 846

Romani, R. W., \& Yadigaroglu, I.-A. 1995, ApJ, 438, 314

Ruderman, M. 1991, ApJ, 366, 261

Ruderman, M., \& Cheng, K. S. 1988, ApJ, 335, 306

Spitkovsky, A. 2006, ApJL, 648, 51

Takata, J., Ng, C. W., \& Cheng, K. S. 2016, MNRAS, 455, 4249

Takata, J., Wang, Y., \& Cheng, K. S. 2010, ApJ, 715, 1318

Takata, J., Wang, Y., \& Cheng, K. S. 2011, MNRAS, 415, 1827

Timokhin, A. N., \& Harding, A. K. 2015, ApJ, 810, 144

Trepl, L., Hui, C. Y., Cheng, K. S., et al. 2010, MNRAS, 405, 1339

Wang, Y., Takata, J., \& Cheng, K. S. 2010, ApJ, 720, 178

Weisskopf, M. C., Romani, R. W., Razzano, M., et al. 2011, ApJ, 743, 74

Zane, S., \& Turolla, R. 2006, MNRAS, 366, 727

Zavlin, V. E., Shibanov, Y. A., \& Pavlov, G. G. 1995, AstL, 21, 149

Zhang, L., \& Cheng, K. S. 1997, ApJ, 480, 370

Zhao, J., Ng, C. W., Lin, L. C. C., et al. 2017, ApJ, 842, 53 